\title{
Performance Analysis on Multiple Device Connections of Small Office Home Office Network
}

\author{
Muhamad Nur Ashaari ${ }^{1} \quad$ Murizah Kassim ${ }^{1,2 *}$ (iD Ruhani Ab. Rahman ${ }^{1}$ Abd Razak Mahmud \\ ${ }^{1}$ School of Electrical Engineering, College of Engineering, Universiti Teknologi MARA, 40450 UiTM Shah Alam, \\ Selangor, Malaysia. \\ ${ }^{2}$ Institute for Big Data Analytics and Artificial Intelligence (IBDAAI), Kompleks Al-Khawarizmi, Universiti Teknologi \\ MARA, 40450 Shah Alam, Selangor, Malaysia. \\ *Corresponding author: murizah@uitm.edu.my \\ E-mails: mnurashaari812@ gmail.com, ruhani467@uitm.edu.my, arazak166@uitm.edu.my
}

Received 18/10/2021, Accepted 14/11/2021, Published 20/12/2021

This work is licensed under a Creative Commons Attribution 4.0 International License.

\begin{abstract}
:
Malaysia has been supported by one of the high-speed fiber internet connections called TM UniFi. TM UniFi is very familiar to be used as a medium to apply Small Office Home Office (SOHO) concept due to the COVID-19 pandemic. Most of the communication vendors offer varieties of network services to fulfill customers' needs and satisfaction during the pandemic. Quality of Services is queried by most users by the fact of increased on users from time to time. Therefore, it is crucial to know the network performance contrary to the number of devices connected to the TM UniFi network. The main objective of this research is to analyze TM UniFi performance with the impact of multiple device connections or users' services. The study was conducted to analyze the QoS on its traffic, packets transfer, RTT, latency, and throughput. Wireshark simulation program has been used as a network traffic capture where PCAP files have been analyzed by using PCAP Analyzer for Splunk. Traffic filtering has been enabled to capture selected traffic to measure network performance. The result shows that better network performance can be achieved if a smaller number of devices are connected at the same time. The percentage of packet loss, RTT, latency is increased when more users connected at the same time. The throughput also shows a decrease for multidevice connections. Based on the analysis it can be concluded that TM UniFi still can provide good network services for the SOHO network environment and sufficient bandwidth despite the rapid user growth in Malaysia.
\end{abstract}

Keywords: Latency, Packet Loss, Quality of Services, RTT, SOHO, Throughput, UniFi Network.

\section{Introduction:}

Technology nowadays is developed rapidly and almost everybody has their computer which allows them to communicate with each other. Computer prices have decreased with the advancement of technology. This enabled individual homeowners to have two or more computers within their place or home office. Therefore, a lot of computer and communication vendors are offering a variety of form of network services that enable the computer to integrate with another computer, share files, an application such as printers, scanner, televisions, and others. The small network computer connected within a household is known as Small Office Home Office (SOHO) networking ${ }^{1,2}$. One of the communication vendors which is Telekom Malaysia (TM) has brought the SOHO concept to one of its services which is TM UniFi. This service has become in-demand work or the home environment because of Internet-based technologies as well as based on current technology Internet of Thing (IoT) and 5G concept. The most important needs are to share the internet link and computing resources such as printers, fax, and storage devices generally are computers on multiple LAN segments but a single IP subnet ${ }^{3}$.

An integrated communication control device integrates the telecommunication environment of a small area with an interface to the network ${ }^{4}$. A device is a stand-alone unit that can be controlled by the user based on the equipment within the office/home. The device is utilized to control integrate device communication within the 
office/home. SOHO system allows the device to communicate with each other. This research has presented a network where the TM UniFi router acted as a medium to allow all equipment in the SOHO environment to communicate in one network. Today's TM UniFi offers services with higher bandwidth for SOHO networks and cheaper solutions. Thus, it is important to ensure whether the bandwidth offered by TM UniFi is optimized by paid services. The services for the network performance have to be transparent to users in achieving its best performance including energy efficiency ${ }^{5}$. High-speed broadband is defined as a bundle of triple-play services that offers three services called High Speed of Internet (HIS), HyppTV (IPTV) and Voice Over Protocol (VOIP) ${ }^{6}$. TM UniFi has provided all features to set up a network for the SOHO concept, but the impact of users' traffic within the network needs to be analyzed and prioritized. Security is one of the important elements to ensure the network is secured. Data usage must be utilized by users and prevent other outsiders come into the network. The SOHO network performance can be evaluated by analysis of the Quality of Services (QoS). QoS is the indicator to measure the level of performance and manage the traffic priority as well as resource control mechanisms ${ }^{7}$. The parameter that QoS analyze is throughput and delay ${ }^{8}$.

Telekom Malaysia Berhad (TM) is the largest integrated communication solutions provider in Malaysia and one of Asia's leading telecommunication companies. TM deals with a broad range of communication essential services solutions in fixed-line, data, and broadband. The SVP framework discovers the primary causes of the decrease in revenue growth rates. Malaysia's household broadband access rate grew from 15.2\% (2007) to $67.1 \%$ (2013). Meanwhile, the growth rate for UniFi, offered by Telekom Malaysia (TM), decreased from $104 \%$ (2012) to $30 \%(2013)^{9}$. A study on the development of IPTV implementation by TM UniFi has been done. The characteristics of Internet Protocol Television (IPTV) have been studied, and this study focuses on its architecture, basic operations, and IPTV protocols itself. The operation of this system is fully generated by Internet Protocol (IP) based on Access Network System. The research presents the IPTV which has been provided by TM UniFi that shows that this service gives good reception to the subscribers even in bad weather (6). An analysis of Skype application network traffic on the Home TM UniFi network also has been studied. It is identified that Skype is one of the most online video media applications used in today's network communication. Previously, Skype is using a telephone line where it is very costly. Today, the UniFi broadband network gives better bandwidth for Skype users and a cheaper solution. However, it is important to determine whether the bandwidth offered by UniFi is fully utilized ${ }^{10}$.

The main objective of this paper is to study and analyze TM UniFi traffic performance and its impact on users' services. The method consists of data collection on a home TM UniFi network using security onion as its base. Wireshark Software has been used to collect PCAP files where Splunk is used as a platform to visualize the data and the PCAP analyzer for Splunk is used to analyze the data collection ${ }^{11}$. Series of experimental work have been carried out for three different environments which are the first by using 2 devices that are connected to the TM UniFi. The second environment is by using 4 devices that are connected, and the third environment is using 6 connected devices. In each environment, data were collected within 15 minutes. The Splunk platform will interface that data and the PCAP analyzer for Splunk will visualize and analyze the data collection. The data were compared to see the impact number of devices connected to TM UniFi performance. The result presents analyzed data on Packet loss, RTT for the packet, latency, and throughput on bandwidth used in the TM UniFi Network. Raw data is then imported into Splunk as a platform. The data were analyzed through PCAP Analyzer for Splunk ${ }^{12}$.

\section{Methodology:}

The methodology consists of experiment setup on network architecture, data collections using Wireshark tools, Splunk, and analyze network performance using PCAP Analyzer for Splunk.

\section{Network Architecture:}

Figure. 1 shows network architecture for the experiment on data captured for network performance on TM UniFi Network. The data of the network traffic are captured by manipulating the number of devices connected to the TM UniFi network, which are 2, 4, and 6 devices connected as per Table 1. The time taken for data collection is within 15 minutes. The UniFi Home Plan that was tested was TM UniFi + Media Box which is RM 158.00 per month as per Table 1 . 


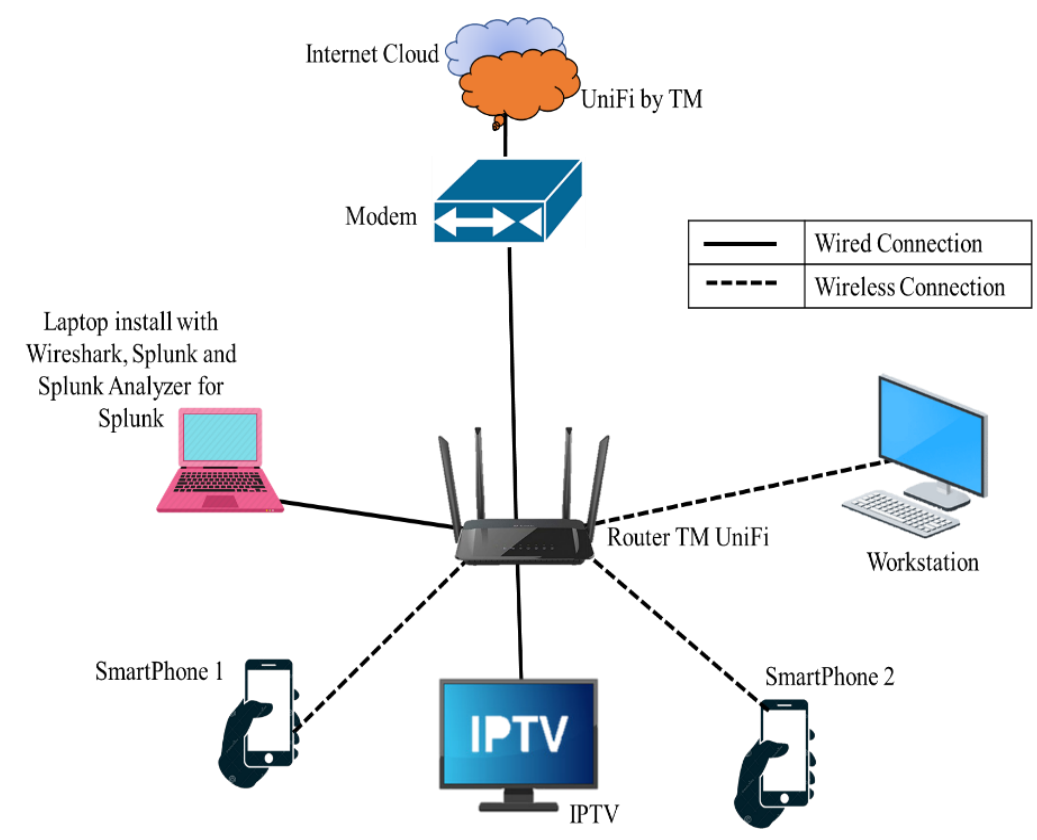

Figure 1. Network Architecture

Table 1. Device Connected

\begin{tabular}{cll}
\hline $\begin{array}{c}\text { Number of Devices } \\
\text { Connected }\end{array}$ & & Devices Connected \\
\hline 2 & $\bullet$ & IPTV \\
& - & Laptop 1 \\
4 & IPTV \\
& - & Laptop 1 \\
& Laptop 2 \\
& Smart Phone 1 \\
6 & IPTV \\
& - & Laptop 1 \\
& Laptop 2 \\
& Smart Phone 1 & Smart Phone 2 \\
& - Smart Phone 3 \\
\hline
\end{tabular}

The device is connected to the TM UniFi network as Table 2 . The price for the download and upload speed is described based on the bandwidth subscribed to the UniFi broadband network.

\section{Table 2. Unifi with media box}

\begin{tabular}{ll}
\hline Price & RM 158.00/month \\
\hline Download speed & Up to 100Mbps \\
Upload speed & Up to 50Mbps \\
Download & Unlimited \\
\hline
\end{tabular}

\section{Data Collection Flow Chart:}

Figure. 2 shows the flowchart system for this research. A scenario is a setup where the device is connected to the TM UniFi. While in this process, Wireshark started to capture network traffic in 15 minutes. The step is repeated for the next connection devices. The device connected is set to 2 devices, 4 devices, and 6 devices simultaneously with capturing in Wireshark. After getting all the data, RTT and packet loss is determined by using PCAP Analyzer for Splunk.

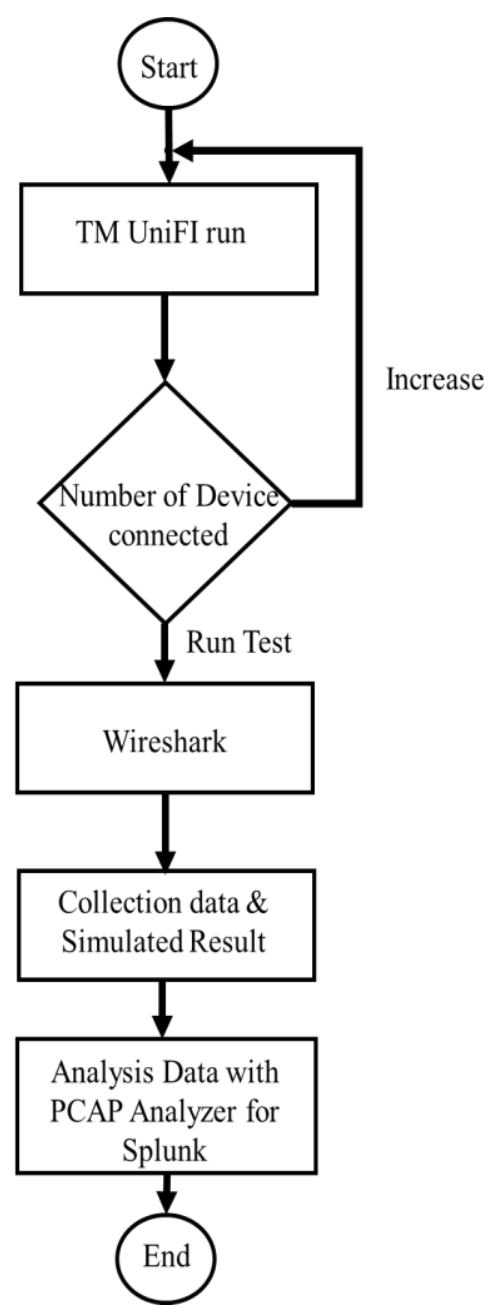

Figure 2. System Flowchart 


\section{Result And Discussion:}

Packet Loss in TCP

Figure. 3 to Figure. 5 show percentage packet loss by PCAP Analyzer for Splunk. It shows packet loss presents for TCP while using PCAP Analyzer for Splunk ${ }^{13}$.

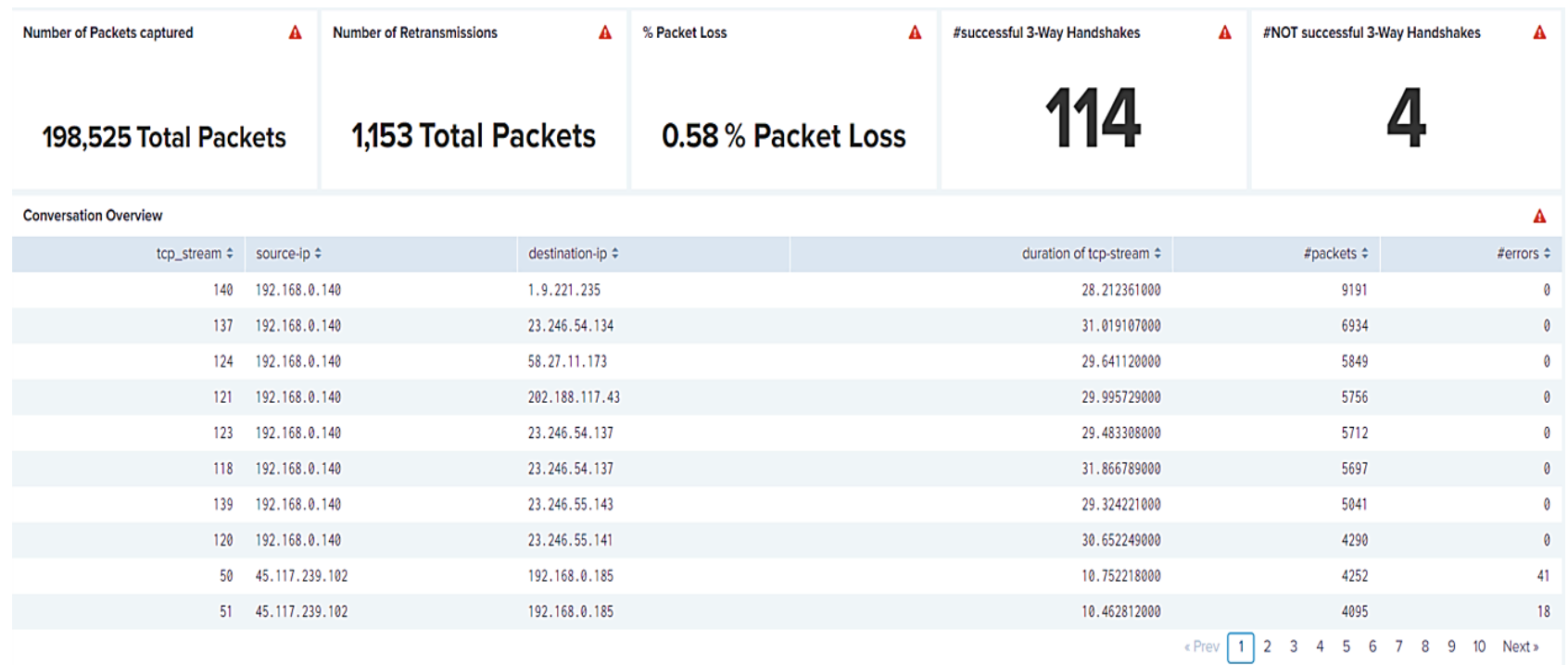

Figure 3. Two Device Connected

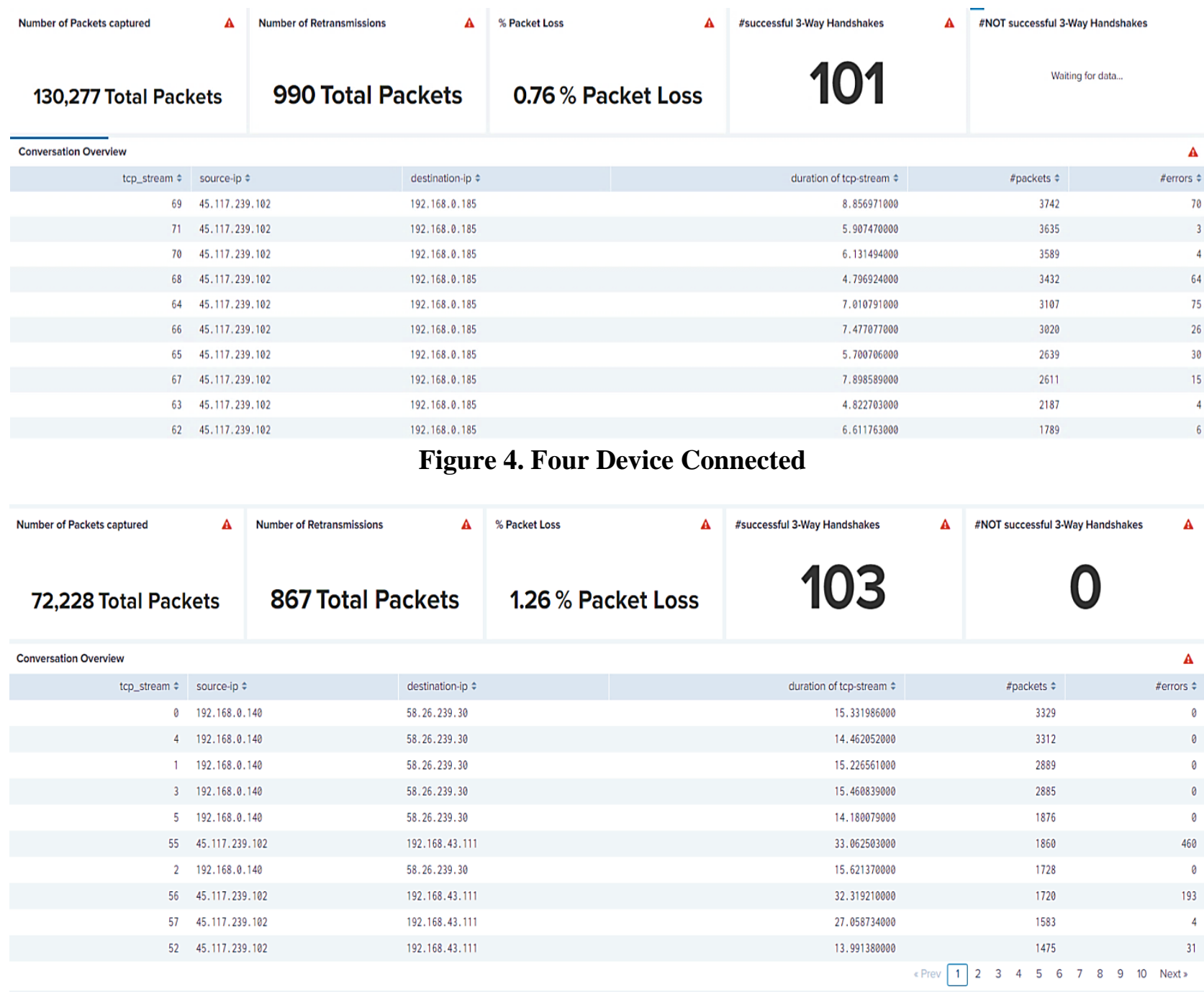

Figure 5. Six Device Connected 
Table 3 presents the percentage Packet Loss analyzer by PCAP Analyzer for Splunk. It is identified that the highest packet loss is $1.26 \%$ which is 6 devices connected to the network within 15 minutes. The lowest packet loss is $0.58 \%$ which is 2 devices connected to the network. The highest packet loss is 867 from 72,226 total packets of data Figure. 3.

\section{Table 3. Percentage Packet Loss}

\begin{tabular}{rrr}
\hline $\begin{array}{l}\text { Number of } \\
\text { Connected }\end{array}$ & Devices & $\begin{array}{l}\text { Percentage of Packet Loss } \\
(\%)\end{array}$ \\
\hline 2 & & 0.58 \\
4 & & \\
6 & & 0.76 \\
\end{tabular}

\section{Round Trip Time (RTT)}

Round Trip Time is the time taken for a signal to travel from signal to be sent until the signal to be acknowledged and received. This time delay consists of the propagation times between the two points of the signal. i. 6 shows overall RoundTrip Time (RTT) for the network with the different number of devices connected and being analyzed by PCAP Analyzer for Splunk. Then being summarized in Table 4. The statistic shows the time delay increase with the number of device increase ${ }^{14}$.

\section{RTT}

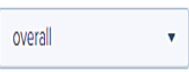

Maximum Round Trip Time

0.75

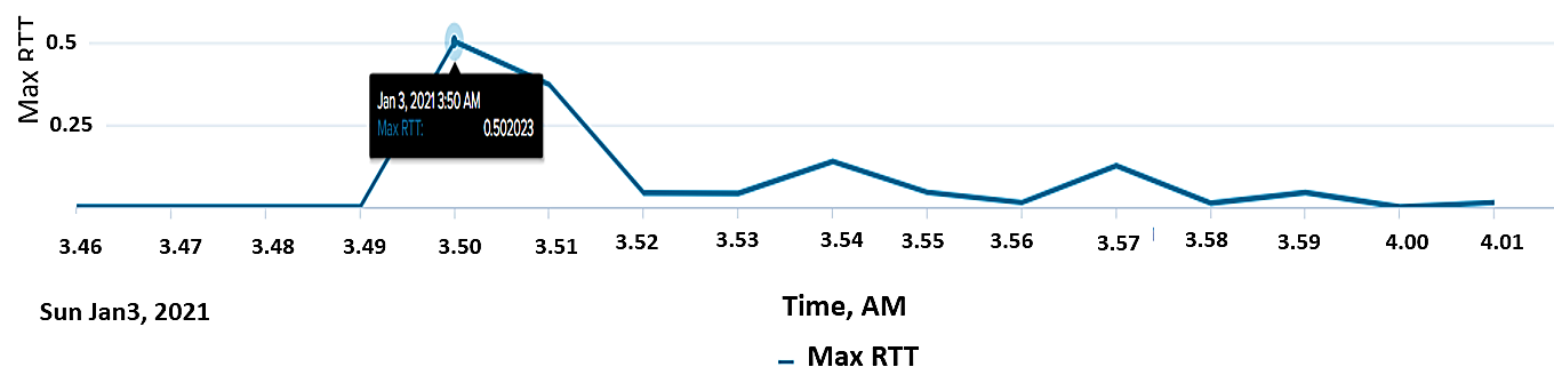

Figure 6. Two Device Connected

RTT

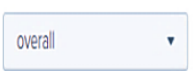

Maximum Round Trip Time

0.75

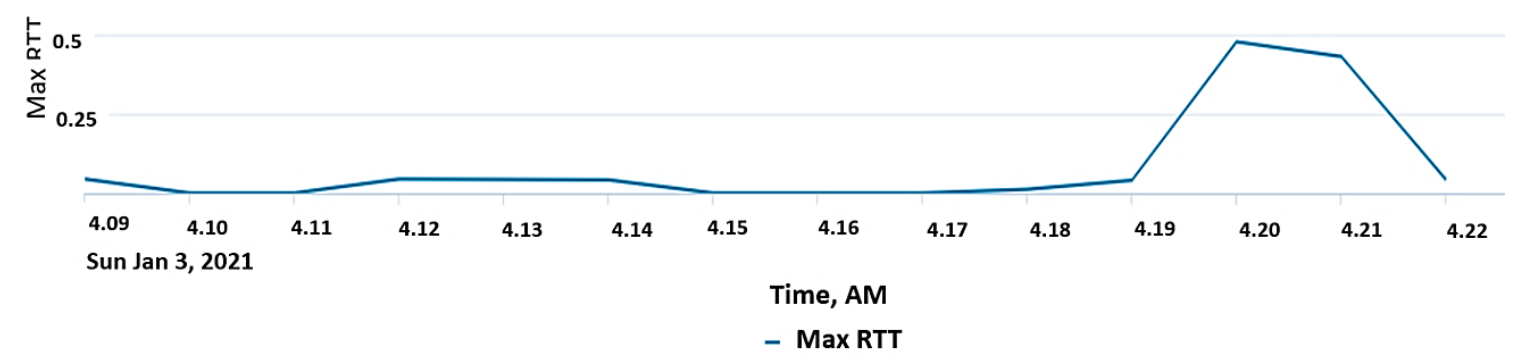

Figure 7. Four Device Connected 


\section{RTT}

overall

Maximum Round Trip Time

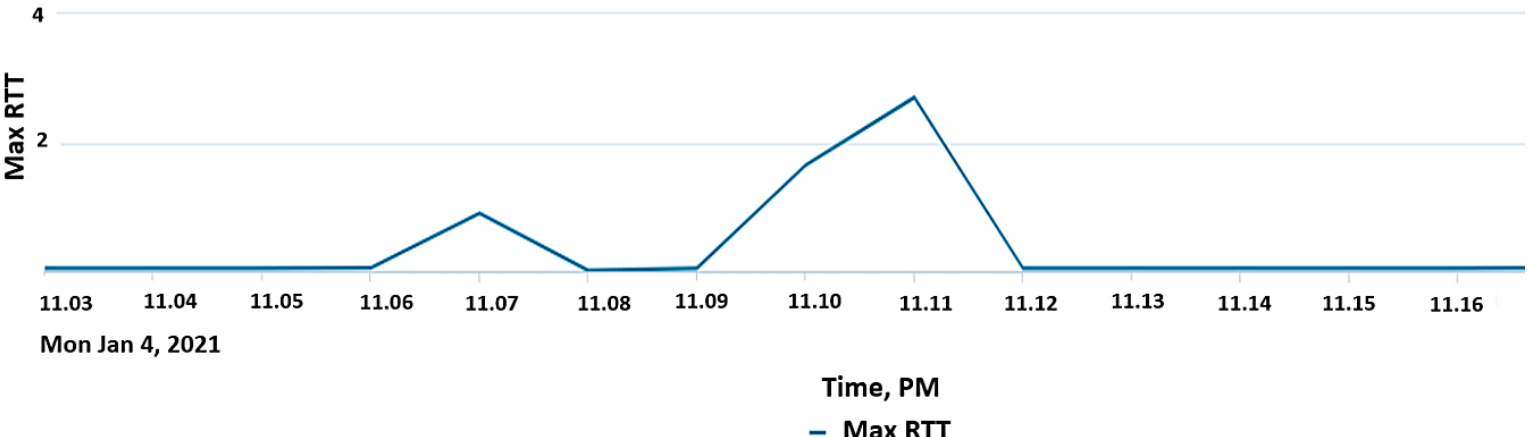

Figure 8. Six Device Connected

Table 4. Average RTT of The Network

\begin{tabular}{cc}
\hline $\begin{array}{c}\text { Number of Devices } \\
\text { Connected }\end{array}$ & Average RTT (ms) \\
\hline 2 & 0.1241 \\
4 & 0.3345 \\
6 & 0.5076 \\
\hline
\end{tabular}

\section{Latency (Delay)}

Network latency is a way to decide to optimize application performance. It is related to RTT of packet data for example high RTT will slow down the application event in a small amount of transmitted data or by addition bandwidth does not improve the throughput when the RTT exceeds the critical point ${ }^{15}$. Table 5 shows the latency of the network with different devices connected. It shows the high number of devices connected, hasa high RTT, hasa high delay time (latency) of $0.16725 \mathrm{~ms}$. The latency was identified by applying the latency Formula as Equation 1.

Latency $=($ Ave $\mathrm{RTT}$ for packets)/2 (ms)

Eq.1

\section{Table 5. Network Latency}

\begin{tabular}{cll}
\hline $\begin{array}{l}\text { Number of } \\
\text { Devices } \\
\text { Connected }\end{array}$ & $\begin{array}{l}\text { Average RTT } \\
(\mathrm{ms})\end{array}$ & Latency $(m s)$ \\
\hline 2 & 0.1241 & 0.06205 \\
4 & 0.3345 & 0.16725 \\
6 & 0.5076 & 0.2638 \\
\hline
\end{tabular}

\section{Throughput:}

Throughput is defined as an actual amount of data that passes through a medium by observed transfer rate as limited by the network bandwidth. For a wireless network, the throughput is limited by the speed of the hardware which means reading the data to send. In other words, it is a rate of data transfer from source to destination that can be transmitted over a sufficiently long period, the performance is measured in Mbit/s by applying the formula as Equation 2. Table 6 shows the throughput of the network. The highest device connected to the network produced the lowest throughput.

Throughput $=($ Packet Size $) /$ Latency $($ Mbits/s $)$ Eq. 2

Table 6. Network Throughput

\begin{tabular}{clll}
\hline $\begin{array}{l}\text { Number of } \\
\text { Devices } \\
\text { Connected }\end{array}$ & $\begin{array}{l}\text { Packet } \\
\text { Size }\end{array}$ & $\begin{array}{l}\text { Latency } \\
(m s)\end{array}$ & $\begin{array}{l}\text { Throughput } \\
\text { (Mbits/s) }\end{array}$ \\
\hline 2 & 198,525 & 0.06205 & 3.1994 \\
4 & 130,277 & 0.16725 & 0.7789 \\
6 & 72,228 & 0.2638 & 0.2738 \\
\hline
\end{tabular}

\section{Conclusion:}

In this research, we investigate the performance of TM UniFi to apply or use as a medium network for the SOHO concept. The number of devices connected to the network has a significant impact on network performance. Parameters such as RTT, total packet, latency, and throughput can be used to analyze network performance. This research has shown that better network performance can be achieved if a smaller number of devices are connected at the same time. It can be concluded that $\mathrm{TM}$ UniFi can provide good network services for the SOHO network environment but needs to control the number of devices connected for best performance. The package TM Unifi needs are wisely chosen due to a load of SOHO because the number of devices connected will give an impact on the network performance. In the future, multiple types of 
services and data can be tested by using Wireshark and PCAP Analyzer for Splunk to identify the network performance. The type of data used could be concentrated on video, voice, or only data transmission which could be analyzed which data type used the most bandwidth at a time.

\section{Acknowledgment:}

The authors would like to thank the College of Engineering, Universiti Teknologi MARA, UiTM Shah Alam for the support grants in publishing this research.

\section{Authors' declaration:}

- Conflicts of Interest: None.

- We hereby confirm that all the Figures and Tables in the manuscript are mine ours. Besides, the Figures and images, which are not mine ours, have been given the permission for republication attached with the manuscript.

- Ethical Clearance: The project was approved by the local ethical committee in Universiti Teknologi MARA.

\section{Authors' contributions:}

M. Kassim conceived of the presented idea. R Ab. Rahman performed the computations. A.R. Mahmud verified the analytical methods. R. Ab. Rahman encouraged M.N Ashaari to investigate the network setup and supervised the findings of this work. All authors discussed the results and contributed to the final manuscript.

\section{References:}

1. Sang J. Hands-on laboratory experiments with SOHO networking technologies. Comput. Appl. Eng. Educ. 2013;21(4):586-95.

2. Syafei WA, Soetrisno YAA, Prasetijo AB, editors. Simple Smart Algorithm for Flexibility of Dynamic Allocation in DHCP Server for SOHO Wireless Router. 2020 International Conference on Computer Engineering, Network, and Intelligent Multimedia (CENIM); 2020 17-18 Nov. 2020.

3. Yu JT, Liu C, editors. Applications and performance analysis of bridging with Layer-3 forwarding on wireless LANs. Advanced Int'l Conference on Telecommunications and Int'l Conference on Internet and Web Applications and Services (AICT-ICIW'06); 2006: IEEE.

4. Kariuki RK. Small Office Home Office WLAN Security Framework. University of Nairobi Research: University of Nairobi; 2012.p 92.

5. Abella CS, Bonina S, Cucuccio A, D'Angelo S, Giustolisi G, Grasso AD, et al. Autonomous energyefficient wireless sensor network platform for home/office automation. IEEE Sens. J. 2019;19(9):3501-12.
6. Sidik N, Abdullah NF, Yusof AL, editors. The development of IPTV implementation by TM UNifi. 2012 International Symposium on Computer Applications and Industrial Electronics (ISCAIE); 2012: IEEE.

7. Ghafar AA, Kassim M, Ya'acob N, Mohamad R, Rahman RA. Qos of wi-fi performance based on signal strength and channel for indoor campus network. BEEI. 2020;9(5):2097-108.

8. Idris MFBM, Yusof MI, Azmat FH, Zain ZM, Rahman RA, Murizah K, editors. Broadband Internet performance (QoS measurement) view from home access gateway in Malaysia. Proceedings - 2014 5th IEEE Control and System Graduate Research Colloquium, ICSGRC 2014; 2014.

9. Ali NNK, Soon BY, Goh LS, Razi NAA. Symptoms versus problems (SVP) in household high speed broadband (HSBB): regaining momentum for Unifi, Malaysia. Probl. Perspect. Manag. 2015(13, Iss. 2 (spec. iss.)):330-46.

10. Kassim M, Ramle SF, Rahman RA, Yusof MI, editors. Skype multimedia application traffic analysis on home Unifi network. ISCAIE 2017 - 2017 IEEE Symposium on Computer Applications and Industrial Electronics; 2017.

11. Carasso D. Exploring splunk: CITO Research New York, USA; 2012.

12. Zhang P. Improving end-to-end quality of service in low-power wireless sensor networks: Universität Oldenburg; 2020.

13. Bruzzese R. An Analysis of Application Logs with Splunk: developing an App for the synthetic analysis of data and security incidents. arXiv preprint arXiv:191211283. 2019.

14. But J, Keller U, Kennedy D, Armitage G, editors. Passive TCP stream estimation of RTT and jitter parameters. The IEEE Conference on Local Computer Networks 30th Anniversary (LCN'05) 1; 2005: IEEE.

15. Randhawa TK. Network Impact Modeling and Analysis: A QoS Perspective. 2020. 


\section{تحليل الأداء على اتصالات الأجهزة المتعددة لثبكة المكاتب الصغيرة بالمنزل \\ محمد نور الأشعري1}

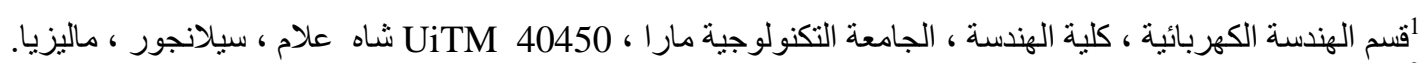

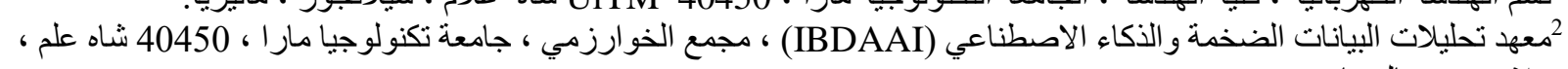
سيلانجور ، ماليزيا.

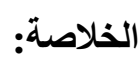

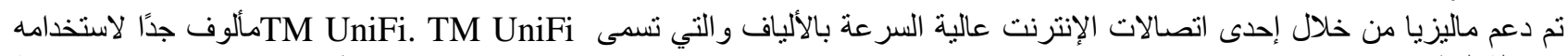

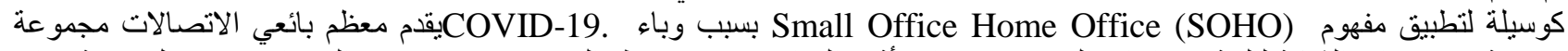

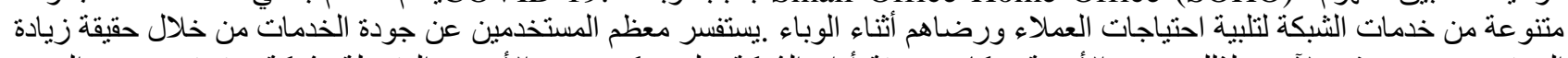

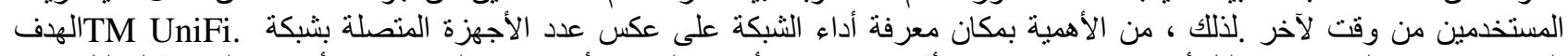

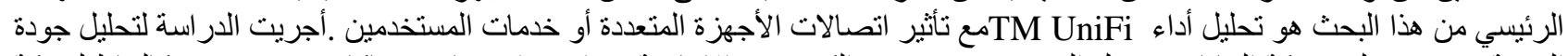

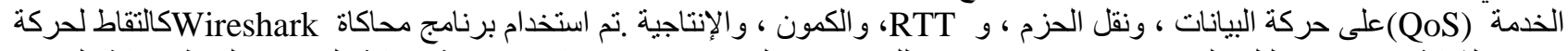

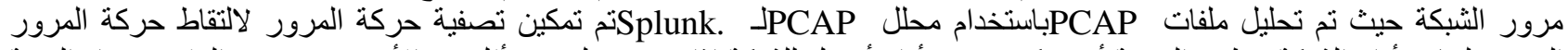

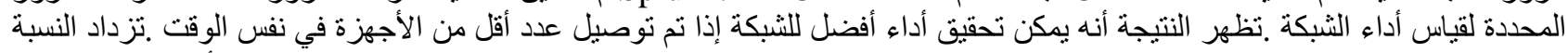

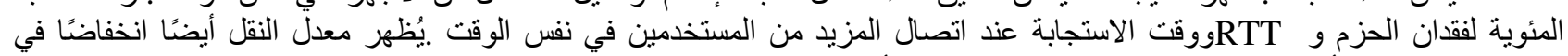

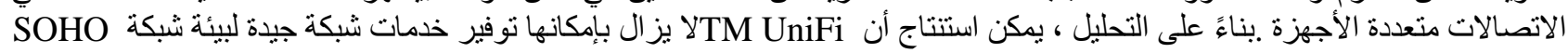
وعرض نطاق ترددي كافٍ على الرغم من النمو السريع للمستخدمين في ماليزيا.

الكلمات المفتاحية: وقت الاستجابة، فقدان الحزمة، جودة الخدمات، SOHO STT، الانتاجية، شبكة UniFi . 